\title{
True polar wander associated with continental drift on a hypothetical Earth
}

\author{
Masao Nakada \\ Department of Earth and Planetary Sciences, Faculty of Science, Kyushu University, Fukuoka 812-8581, Japan
}

(Received May 23, 2006; Revised March 17, 2007; Accepted April 11, 2007; Online published June 27, 2007)

\begin{abstract}
Long-term true polar wander of the Earth (TPW) has generally been discussed by taking into account convective processes in the mantle such as downgoing slabs and upwelling plumes. Here I examined a relationship between continental drift and TPW on a hypothetical Earth with no such convective processes in the mantle. I evaluated temporal changes in moments of inertia owing to continental drift during a period of $\sim 250$ Ma based on a paleogeographic reconstruction, in which I estimated the lateral density heterogeneities by factoring in the observed mean land elevation of continents and average age of the oceanic lithosphere. The predictions for a viscoelastic Earth model with plausible viscosity models indicate that the long-term TPW might have been affected by continental drift throughout Cenozoic and Mesozoic times, which has wholly proceeded by maintaining isostasy at a certain depth, as well as convective processes in the mantle.
\end{abstract}

Key words: Earth's rotation, true polar wander, continental drift, Maxwell viscoelasticity, viscosity, Love number.

\section{Introduction}

Time-dependent mass redistribution of the Earth and its related deformation cause temporal changes of the Earth's rotation axis-i.e. true polar wander (TPW) (e.g., Munk and MacDonald, 1960; Lambeck, 1980). Recent geodetic data indicate TPW at $\sim 1^{\circ} \mathrm{Myr}^{-1}$ towards Greenland (Dickman, 1977; McCarthy and Luzum, 1996), which has been generally interpreted in terms of the glacio- hydro-isostatic adjustment associated with the Quaternary glacial cycles (e.g., Sabadini and Peltier, 1981; Wu and Peltier, 1984; Mitrovica and Milne, 1998; Johnston and Lambeck, 1999; Nakada, 2002; Nakada and Okuno, 2003; Mitrovica et al., 2005).

The long-term polar wander throughout geologic time has been derived from paleomagnetic data, based on the assumption of hotspot fixity (e.g., Besse and Courtillot, 2002). However, the assumption is controversial (e.g., Tarduno et al., 2003). Figure 1 illustrates the TPW path during the past approximately 200 Myr obtained by Besse and Courtillot (2002) under the hypothesis of fixed Atlantic and Indian hotspots, which these authors referred to as BC01 as denoted by them. In this figure, $95 \%$ confidence intervals (Besse and Courtillot, 2002) are not shown for the purpose of clarity. The general features for BC01 are similar to those reported by Besse and Courtillot (1991) and Prévot et al. (2000) (Besse and Courtillot, 2002). Although the behavior of TPW prior to $130 \mathrm{Ma}$ is less reliable compared with that after $130 \mathrm{Ma}$ because of the uncertainties in the models of hotspot kinematics, Besse and Courtillot (2002) indicated several important features of the observed TPW path. One is that TPW is episodic, with periods of quasi-standstill al-

Copyright (c) The Society of Geomagnetism and Earth, Planetary and Space Sciences (SGEPSS); The Seismological Society of Japan; The Volcanological Society of Japan; The Geodetic Society of Japan; The Japanese Society for Planetary Sciences; TERRAPUB. ternating with periods of faster TPW in the Cretaceous. The periods of quasi-standstill, with polar wander speeds generally less than $0.2^{\circ} \mathrm{Myr}^{-1}$, are 160-130 Ma and 50-10 Ma, while the period spanning 130-60 Ma has relatively faster polar wander speeds of $0.3^{\circ}-0.5^{\circ} \mathrm{Myr}^{-1}$. The second feature is that the direction of TPW for about 130-50 Ma is nearly opposite to that of the past $\sim 40 \mathrm{Myr}$, with the rotation axis showing a drift towards Greenland over these past $\sim 40$ Myr, similar to the direction obtained by the geodetic data (Dickman, 1977; McCarthy and Luzum, 1996). Besse and Courtillot (2002) also compared TPW for Atlantic and Indian hotspots with that for Pacific hotspots only and indicated that TPW is a truly global feature of Earth dynamics.

The causes of long-term polar wander are classical but fundamental problems for geoscientists and have been topics of discussion for many years (e.g., Gold, 1955; Munk and MacDonald, 1960; Goldreich and Toomre, 1969; Lambeck, 1980). More recently, the long-term polar wander has been quantitatively studied based on lithospheric processes that are unrelated to glacial cycles and which take place under non-isostatic conditions such as mountain building and erosion (Vermeersen and Vlaar, 1993; Vermeersen et al., 1994) and convective processes in the mantle with lateral density heterogeneities due to downgoing slabs and upwelling plumes (Ricard and Sabadini, 1990; Spada et al., 1992; Ricard et al., 1993; Richards et al., 1997, 1999; Steinberger and O'Connell, 1997, 2002; GreffLefftz, 2004). In fact, convective processes may reasonably explain the observed speeds of TPW-less than $1^{\circ} \mathrm{Myr}^{-1}$ during the Cenozoic and late Mesozoic, whereas the geometry of the forcing is just as much as the Earth model responsible for the fit (e.g., Richards et al., 1997, 1999; Steinberger and O'Connell, 1997, 2002). Steinberger and O'Connell (2002) investigated the TPW path in the Cenozoic time in response to changes in the degree of two non- 


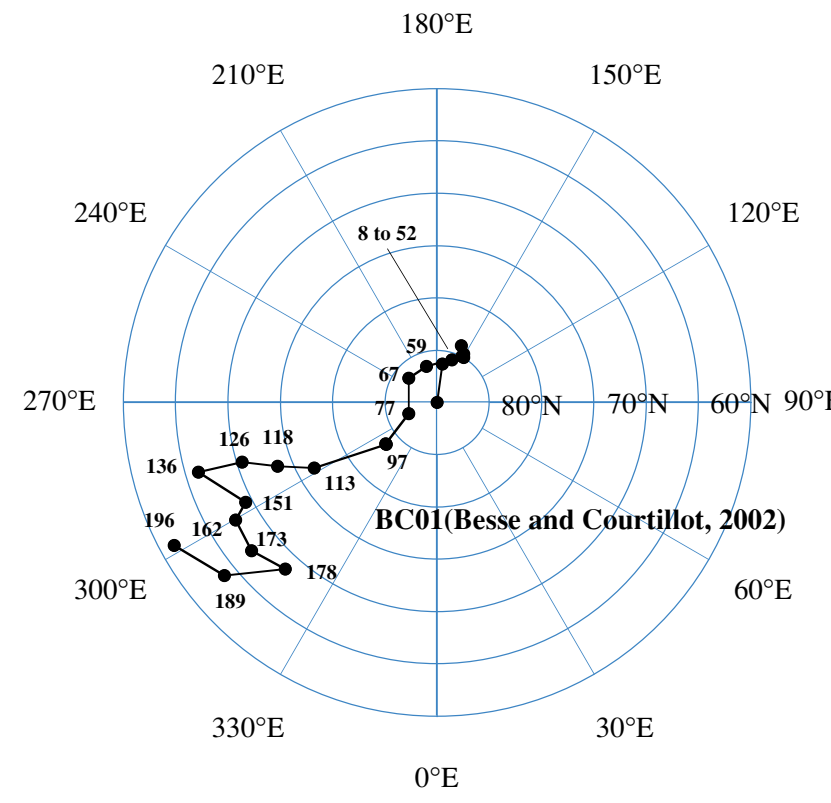

Fig. 1. Observed TPW path during the past $\sim 200 \mathrm{Myr}$ under the hypothesis of fixed Atlantic and India hotspots, referred to here as BC01 (Besse and Courtillot, 2002). Time unit is Ma.

hydrostatic components of the equipotential surface by examining mantle flow models for a number of published tomographic models. They then indicated that the change of the geoid of approximately $-4 \mathrm{~m} \mathrm{Myr}^{-1}$ at the northern tip of New Zealand is required in order to explain the paleomagnetically observed Cenozoic polar wander towards Greenland (e.g., Besse and Courtillot, 2002). That is, the magnitude for the change during the past $60 \mathrm{Myr}$ amounts to $240 \mathrm{~m}$, roughly two- or three-fold larger than that of the geoid anomalies observed at the present-time (Lemoine et al., 1998).

On the other hand, the distribution of continents and oceans is undoubtedly a significant source for the most dominant lateral density heterogeneities of the Earth, as shown, for example, indicated by the facts that the observed land elevation of continents is $875 \mathrm{~m}$ and the present oceanic lithospheres subduct an average age of $\sim 120 \mathrm{Myr}$ (e.g., Turcotte and Schubert, 2002). If we consider that continental drift-i.e. plate movement-approximately proceeds with keeping isostasy at a certain depth (e.g., Turcotte and Schubert, 2002), then continental drift causes significant time-dependent changes in moments of inertia. For example, temporal changes in moments of inertia associated with the breakup of Pangea followed by seafloor spreading of the Atlantic Ocean might have produced nonnegligible effects on TPW during the past 200 Myr. Dickman (1979) discussed the relationship between continental drift and TPW. By employing Airy isostatic compensation, he estimated changes in the components of the inertia tensor when the lithospheric plates have drifted for $1 \mathrm{Myr}$ according to the absolute plate velocity of Minster et al. (1974). He then indicated that these changes induce a polar shift of 0.63 arc seconds for an elastic Earth, which is significantly smaller than the geodetically observed speed of $\sim 1^{\circ} \mathrm{Myr}^{-1}$ (Dickman, 1977; McCarthy and Luzum, 1996). However, it should be noted that the estimate (Dickman, 1979) is a polar shift for 1 Myr for an elastic Earth and that viscoelastic responses of the rotational bulge were not taken into account in estimating polar shift.

Here I examine the effects of continental drift on TPW based on a viscoelastic Earth in which I adopt a hypothetical earth model with no convective processes in the mantle such as downgoing slabs and mantle plumes in the mantle.

\section{Mathematical Formulation}

I examine the long-term polar motion of the rotation axis associated with continental drift based on a Maxwell viscoelastic earth model with an inviscid core. I adopt a compressible Earth model with elasticity and density given by the seismological model PREM (Dziewonski and Anderson, 1981). The angular momentum, $\boldsymbol{H}$, is conserved in the case with no external torque. Then, the Liouville equation describing the Earth's rotational variations in a rotating frame is given by (e.g., Munk and MacDonald, 1960; Lambeck, 1980):

$$
\frac{\partial \boldsymbol{H}}{\partial t}+\boldsymbol{\omega} \times \boldsymbol{H}=0
$$

where $\boldsymbol{H}=\boldsymbol{J} \cdot \boldsymbol{\omega} . \quad \boldsymbol{J}$ is the time-dependent second-degree symmetric inertia tensor and $\boldsymbol{\omega}$ is the angular velocity. The inertia tensor, $J_{i j}(t)$, examined in this study is given by:

$$
\begin{aligned}
J_{i j}(t)= & I \delta_{i j}+\frac{k^{\mathrm{T}}(t) a^{5}}{3 G} *\left[\omega_{i}(t) \omega_{j}(t)-\frac{1}{3} \omega^{2}(t) \delta_{i j}\right] \\
& +\delta(t) * E_{i j}(t)
\end{aligned}
$$

where $\delta_{i j}$ is the Kronecker delta, $a$ is the Earth's radius, $G$ is the gravity constant, $\omega^{2}(t)=\omega_{1}^{2}(t)+\omega_{2}^{2}(t)+\omega_{3}^{2}(t)$, $\delta(t)$ is the delta function and the asterisk denotes a time convolution. $I$ is the inertia tensor for a non-rotating Earth and $E_{i j}(t)$ is the time-dependent moment of inertia due to continental drift.

The second term of Eq. (2) is the inertia tensor associated with the viscoelastic deformation due to the rotational potential and represents the effects of the rotational bulge on TPW (Munk and MacDonald, 1960). This term is given by convolution using degree-two tidal Love number, $k^{\mathrm{T}}(t)$, sensitive to the viscosity structure of the Earth's mantle (Peltier, 1976). In particular, we have to adopt a realistic density structure in evaluating the values of $k^{\mathrm{T}}(t)$ for $t=\infty$ (Nakada, 2002).

The last term is associated with the time-dependent distribution of continents and oceans and is derived from the lateral density heterogeneities between the continents and oceans. In TPW related to the glacio-hydro-isostatic adjustment process (e.g., Sabadini and Peltier, 1981; Wu and Peltier, 1984; Mitrovica and Milne, 1998; Johnston and Lambeck, 1999; Nakada, 2002; Nakada and Okuno, 2003) and the Earth's deformation due to surface loads (Vermeersen and Vlaar, 1993; Vermeersen et al., 1994) and internal loads (Ricard et al., 1993; Steinberger and O'Connell, 1997, 2002; Richards et al., 1997, 1999; GreffLefftz, 2004), the total moments of inertia are contributed by viscoelastic responses to those for the loads $\left(L_{i j}(t)\right)$ 
and consequently given by $\left[\delta(t)+k^{\mathrm{L}}(t)\right] * L_{i j}(t)$ using a degree-two load Love number, $k^{\mathrm{L}}(t)$, that is sensitive to the viscosity structure of the Earth's mantle (e.g., Peltier, 1976). However, the viscoelastic responses for temporal changes in moments of inertia owing to the continental drift may be insignificant because continental drift wholly proceeds by maintaining isostasy at a certain depth.

In Eq. (1), the term of $\partial \boldsymbol{H} / \partial t$ may safely be neglected in an evaluation of the secular term of TPW on geological timescales as for glacial rebound problem (e.g., Wu and Peltier, 1984; Vermeersen and Sabadini, 1996; Mitrovica and Milne, 1998; Nakada, 2002). We then obtain $\omega^{2}=\Omega^{2}$ if we adopt this assumption (e.g., Greff-Lefftz, 2004). $\Omega$ is the sidereal rotation rate. That is, the non-linear Liouville equations to be solved are:

$$
\begin{gathered}
\omega_{2} H_{3}-\omega_{3} H_{2}=0 \\
\omega_{3} H_{1}-\omega_{1} H_{3}=0 \\
\omega_{1}^{2}+\omega_{2}^{2}+\omega_{3}^{2}=\Omega^{2}
\end{gathered}
$$

The degree-two tidal Love number is given by (Peltier, 1974, 1976):

$$
k^{\mathrm{T}}(t)=k^{\mathrm{T}, \mathrm{E}} \delta(t)+\sum_{j=1}^{N} \gamma_{j} e^{s_{j} t}
$$

where $k^{\mathrm{T}, \mathrm{E}}$ is the elastic tidal Love number, $-s_{j}$ is the inverse of relaxation time of eigenmode $j$, and $\gamma_{j}$ is the strength of eigenmode $j$. Substituting Eqs. (2) and (6) into Eq. (3), we get:

$$
\begin{aligned}
\frac{a^{5}}{3 G}\left\{\left[\sum_{j=1}^{N} \gamma_{j} \int_{0}^{t} e^{s_{j}(t-\tau)} \omega_{1}(\tau) \omega_{3}(\tau) d \tau\right] \omega_{1}(t) \omega_{2}(t)\right. \\
\left.-\left[\sum_{j=1}^{N} \gamma_{j} \int_{0}^{t} e^{s_{j}(t-\tau)} \omega_{1}(\tau) \omega_{2}(\tau) d \tau\right] \omega_{1}(t) \omega_{3}(t)\right\} \\
+\frac{a^{5}}{3 G}\left\{\left[\sum_{j=1}^{N} \gamma_{j} \int_{0}^{t} e^{s_{j}(t-\tau)} \omega_{2}(\tau) \omega_{3}(\tau) d \tau\right] \omega_{2}^{2}(t)\right. \\
\left.-\left[\sum_{j=1}^{N} \gamma_{j} \int_{0}^{t} e^{s_{j}(t-\tau)} \omega_{2}^{2}(\tau) d \tau\right] \omega_{2}(t) \omega_{3}(t)\right\} \\
+\frac{a^{5}}{3 G}\left\{\left[\sum_{j=1}^{N} \gamma_{j} \int_{0}^{t} e^{s_{j}(t-\tau)} \omega_{3}^{2}(\tau) d \tau\right] \omega_{2}(t) \omega_{3}(t)\right. \\
\left.-\left[\sum_{j=1}^{N} \gamma_{j} \int_{0}^{t} e^{s_{j}(t-\tau)} \omega_{2}(\tau) \omega_{3}(\tau) d \tau\right] \omega_{3}^{2}(t)\right\} \\
+E_{13}(t) \omega_{1}(t) \omega_{2}(t)-E_{12}(t) \omega_{1}(t) \omega_{3}(t) \\
+E_{23}(t) \omega_{2}^{2}(t)-E_{22}(t) \omega_{2}(t) \omega_{3}(t) \\
+E_{33}(t) \omega_{2}(t) \omega_{3}(t)-E_{23}(t) \omega_{3}^{2}(t)=0
\end{aligned}
$$

Here we define

$$
A_{k l}(n, j)=\int_{0}^{n \Delta t} e^{s_{j}(n \Delta t-\tau)} \omega_{k}(\tau) \omega_{l}(\tau) d \tau
$$

and

$B_{k l}(n, j)=\frac{e^{s_{j} n \Delta t}}{2} \omega_{k}(0) \omega_{l}(0)+\sum_{m=1}^{n-1} e^{s_{j}(n-m) \Delta t} \omega_{k}(m) \omega_{l}(m)$

Then, we get

$$
A_{k l}(n, j)=\left[B_{k l}(n, j)+\frac{1}{2} \omega_{k}(n) \omega_{l}(n)\right] \Delta t
$$

and

$$
B_{k l}(n, j)=\left[B_{k l}(n-1, j)+\omega_{k}(n-1) \omega_{l}(n-1)\right] e^{s_{j} \Delta t}
$$

where $B_{k l}(n-1, j)$ is given by $\omega_{k}(t)$ and $\omega_{l}(t)$ for $t \leq(n-$ 1) $\Delta t$. By using these relationships, we get the following form for $t=n \Delta t$ :

$$
\begin{aligned}
& \sum_{j=1}^{N} \gamma_{j} \int_{0}^{t} e^{s_{j}(t-\tau)} \omega_{k}(\tau) \omega_{l}(\tau) d \tau \\
& =\sum_{j=1}^{N} \gamma_{j}\left[B_{k l}(n, j)+\frac{1}{2} \omega_{k}(n) \omega_{l}(n)\right] \Delta t
\end{aligned}
$$

Substituting Eq. (12) into Eq. (7), Eq. (3) describing the angular velocity at time $n \Delta t$ is expressed in a simple form:

$$
\begin{aligned}
& \frac{a^{5}}{3 G}\left[\left(f_{13} \omega_{1} \omega_{2}-f_{12} \omega_{1} \omega_{3}\right)+\left(f_{23} \omega_{2}^{2}-f_{22} \omega_{2} \omega_{3}\right)\right. \\
& \left.+\left(f_{33} \omega_{2} \omega_{3}-f_{23} \omega_{3}^{2}\right)\right]+\left[\left(E_{13} \omega_{1} \omega_{2}-E_{12} \omega_{1} \omega_{3}\right)\right. \\
& \left.+\left(E_{23} \omega_{2}^{2}-E_{22} \omega_{2} \omega_{3}\right)+\left(E_{33} \omega_{2} \omega_{3}-E_{23} \omega_{3}^{2}\right)\right] \\
& =0
\end{aligned}
$$

where

$$
f_{k l}=\Delta t \sum_{j=1}^{N} \gamma_{j} B_{k l}(n, j)
$$

Similarly, Eq. (4) is given by:

$$
\begin{aligned}
& \frac{a^{5}}{3 G}\left[\left(f_{11} \omega_{1} \omega_{3}-f_{13} \omega_{1}^{2}\right)+\left(f_{12} \omega_{2} \omega_{3}-f_{23} \omega_{1} \omega_{2}\right)\right. \\
& \left.+\left(f_{13} \omega_{3}^{2}-f_{33} \omega_{1} \omega_{3}\right)\right]+\left[\left(E_{11} \omega_{1} \omega_{3}-E_{13} \omega_{1}^{2}\right)\right. \\
& \left.+\left(E_{12} \omega_{2} \omega_{3}-E_{23} \omega_{1} \omega_{2}\right)+\left(E_{13} \omega_{3}^{2}-E_{33} \omega_{1} \omega_{3}\right)\right] \\
& =0
\end{aligned}
$$

We evaluate $\omega_{i}(n)(i=1,2,3)$ from Eqs. (5), (13) and (15) by using an iteration method, and easily get converged solutions for $\Delta t=1$ year and third iteration. The time integrations for these non-linear equations start from $t=0$. In order to evaluate the viscoelastic responses accurately, however, the theoretical lower limit of the integration should be infinity unless the rotation suddenly started at $t=0$. I discuss this point in Section 3.3. 


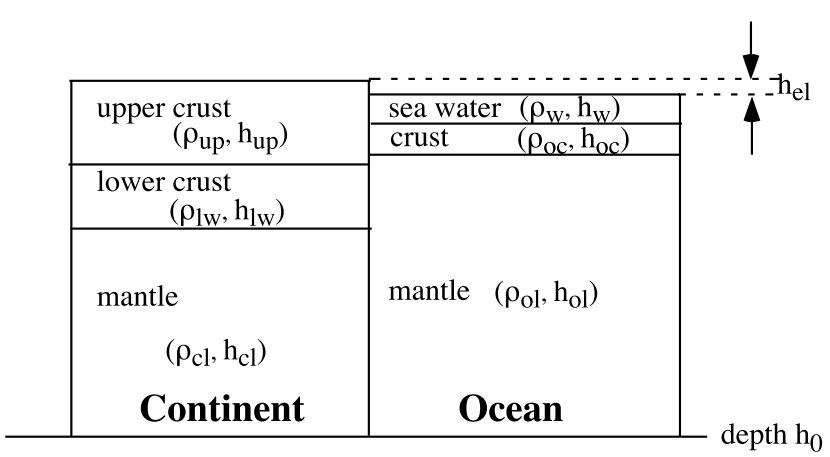

Fig. 2. A schematic figure of density structures for continents and oceans. Isostasy is assumed at a depth $h_{0}$, and the mean land elevation is denoted by $h_{\mathrm{el}}$.

\section{Results and Discussion}

\subsection{Density and viscosity models}

I evaluate TPW due to lateral density heterogeneities resulting from the time-dependent distribution of the continents and oceans. It is assumed that isostasy is kept at a certain depth, $h_{0}$, in the process of continental drift. The mean land elevation is denoted by $h_{\mathrm{el}}$. The vertical density structures of the continents and oceans are denoted by $\rho_{\mathrm{c}}(r)$ and $\rho_{\mathrm{o}}(r)$, respectively, and assumed to be constant within the continents and oceans. A schematic view of density structures for continents and oceans is depicted in Fig. 2. For the continental area above the depth $h_{0}$, we adopt a simple model composed of upper crust, lower crust and mantle part of continental lithosphere. The densities of these layers are denoted by $\rho_{\mathrm{up}}, \rho_{\mathrm{lw}}$, and $\rho_{\mathrm{cl}}$, and the thicknesses are denoted by $h_{\mathrm{up}}, h_{\mathrm{lw}}$, and $h_{\mathrm{cl}}$, respectively. In the ocean area above the depth $h_{0}$, the structure is composed of seawater with density $\rho_{\mathrm{w}}$ and thickness $h_{\mathrm{w}}$, a crust with $\rho_{\mathrm{oc}}$ and $h_{\mathrm{oc}}$, and mantle part of the oceanic lithosphere with $\rho_{\mathrm{ol}}$ and $h_{\mathrm{ol}}$.

We denote the moments of inertia at time $t, C_{i j}(t)$, for the density structure above the depth $h_{\mathrm{o}}$. The value of $E_{i j}(t)$ in Eq. (2) is defined by changes in $C_{i j}(t)$ relative to that for a certain reference time $t_{0}(t=0$ for Eqs. (5), (13) and (15)). That is, $E_{i j}(t)$ is defined by:

$$
E_{i j}(t)=C_{i j}(t)-C_{i j}\left(t_{0}\right)
$$

The form of $C_{i j}(t)$ is given by:

$$
\begin{aligned}
C_{i j}(t)= & \left(F_{c}-F_{o}\right) \int_{C D(t)} K_{i j}(\theta, \varphi) d \theta d \varphi \\
& +F_{o} \int_{w} K_{i j}(\theta, \varphi) d \theta d \varphi
\end{aligned}
$$

where $F_{c}=\int_{a-h_{0}}^{a+h_{\mathrm{el}}} \rho_{c} r^{4} d r, F_{o}=\int_{a-h_{0}}^{a} \rho_{o} r^{4} d r, C D(t)$ indicates the distribution of continents at time $t$ and ' $w$ ' means the whole Earth. $K_{i j}(\theta, \varphi)$ corresponds to each component of moments of inertia. The first term in Eq. (17) only causes the temporal changes on TPW because the second term is time-independent; that is, the form of $E_{i j}(t)$ defined by Eq. (16) is given by:

$$
E_{i j}(t)=\left(F_{c}-F_{o}\right)\left[\int_{C D(t)} K_{i j}(\theta, \varphi) d \theta d \varphi\right.
$$

Table 1. Density structure model used in this study.

\begin{tabular}{crlc}
\hline \multicolumn{2}{c}{ Density $\left(\mathrm{kg} \mathrm{m}^{-3}\right)$} & \multicolumn{2}{c}{ Thickness $(\mathrm{km})$} \\
\hline$\rho_{\mathrm{up}}$ & 2800.0 & $h_{\mathrm{up}}$ & 20.0 \\
$\rho_{\mathrm{lw}}$ & 3150.0 & $h_{\mathrm{lw}}$ & 20.0 \\
$\rho_{\mathrm{cl}}$ & 3300.0 & $h_{\mathrm{cl}}$ & 41.9 \\
$\rho_{\mathrm{w}}$ & 1000.0 & $h_{\mathrm{w}}$ & 5.0 \\
$\rho_{\mathrm{oc}}$ & 2950.0 & $h_{\mathrm{oc}}$ & 6.0 \\
$\rho_{\mathrm{ol}}$ & 3350.0 & $h_{\mathrm{ol}}$ & 70.0 \\
\hline \multicolumn{3}{c}{$h_{\mathrm{el}}(\mathrm{m})$} & \multicolumn{2}{c}{878.8} \\
\hline \multicolumn{2}{c}{$F_{c}-F_{o}\left(\times 10^{32} \mathrm{~kg} \mathrm{~m}^{2}\right)$}
\end{tabular}

$$
\left.-\int_{C D\left(t_{0}\right)} K_{i j}(\theta, \varphi) d \theta d \varphi\right]
$$

As we adopt reference times of $t_{0}=250,210$ and $40 \mathrm{Ma}$ ( $t=0$ for Eqs. (5), (13) and (15)) in evaluating TPW, it is useful to define the first term of Eq. (17) by $D_{i j}(t)$ :

$$
D_{i j}(t)=\left(F_{c}-F_{o}\right) \int_{C D(t)} K_{i j}(\theta, \varphi) d \theta d \varphi
$$

The density structure model adopted here is given in Table 1. These density structures are derived from the PREM (Dziewonski and Anderson, 1981), a mean land elevation with $875 \mathrm{~m}$ (e.g., Turcotte and Schubert, 2002) and also by considering that the present oceanic lithospheres subduct at an average age of $\sim 120$ Myr (e.g., Turcotte and Schubert, 2002). The isostasy is assumed to be a depth of $81 \mathrm{~km}$. The density model is adopted only for evaluating the changes in moments of inertia associated with continental drift, and I examine TPW by adopting several lithospheric thicknesses. The value of $F_{c}-F_{o}$ for this model is $3.02 \times 10^{32} \mathrm{~kg} \mathrm{~m}^{2}$.

I next explain the viscosity models required to evaluate the tidal Love number, $k^{\mathrm{T}}(t)$, for a Maxwell viscoelastic Earth model. A realistic rheological structure for the lithosphere is adopted for examining the lithospheric response with geological timescales (e.g., Watts, 2001). I therefore employ an earth model with a depth-dependent lithospheric viscosity defined by $\eta(D)=\eta_{\text {ltop }} 10^{-\Delta \eta D / H}$, where $D$ is the depth from the surface, $H$ is the lithospheric thickness, $\eta_{\text {ltop }}$ is the viscosity at the surface, $\Delta \eta=\log \left(\eta_{\text {ltop }} / \eta_{\text {um }}\right)$ and $\eta_{\mathrm{um}}$ is the upper mantle viscosity above a depth of $670 \mathrm{~km}$ (Nakada, 2002). In this study, we adopt $H=100$ and $200 \mathrm{~km}$ and $\eta_{\text {ltop }}=10^{22}, 10^{24}$, and $10^{26} \mathrm{~Pa} \mathrm{~s}$ for these parameters, whereas the viscosity structures of the lithospheres remain controversial despite extensive studies (e.g., Watts, 2001). The predictions with $H=100 \mathrm{~km}$ are nearly the same as those for $H=81 \mathrm{~km}$. The upper mantle viscosity, which is less sensitive to the prediction in a plausible range of $10^{20}<\eta_{\text {um }}<10^{21} \mathrm{~Pa}$ s (e.g., Nakada and Lambeck, 1989; Mitrovica and Forte, 2004), is assumed to be $10^{21} \mathrm{~Pa} \mathrm{~s}$. The lower mantle viscosity from $670 \mathrm{~km}$ depth to the core-mantle boundary is denoted by $\eta_{\mathrm{lm}}$.

\subsection{Continental distributions and moments of inertia}

In this study, I calculate TPW based on two different continental distribution models. One is a realistic model and the other is a hypothetical model discussed below. In order 

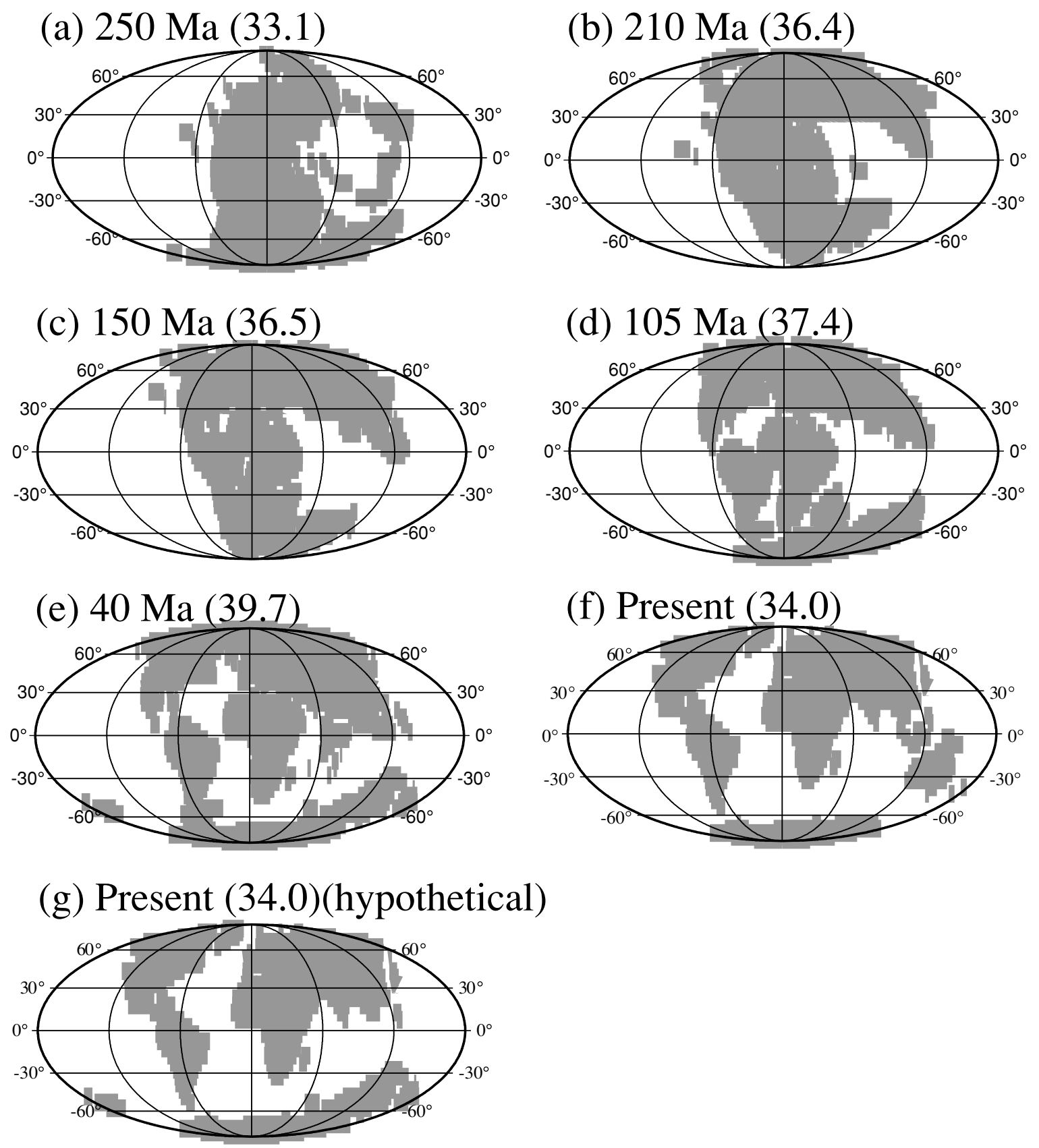

Fig. 3. (a)-(f) Paleogeographic continental distributions at each time point reconstructed by Condie and Sloan (1997). (g) A hypothetical model for the distribution of continents and oceans at the present time. The continent including Australia at 40 Ma is fixed during the past 40 Myr and the distribution of other continents is the same as that for the present one. The number within the parenthesis indicates the percentage of the continental area to the area of the whole Earth, $A C(t)$.

to estimate temporal changes in moments of inertia due to continental drift, I adopt a paleogeographic reconstruction by Condie and Sloan (1997) as a possible model. Figure 3 illustrates the distributions of continents and oceans at 250 , $210,150,105$, and $40 \mathrm{Ma}$ and the present time (Condie and Sloan, 1997). There is no doubt that there are a number of uncertainties in the reconstructions, particularly before $\sim 130 \mathrm{Ma}$, which provide little information for paleomagnetic results with reliable hotspot kinematics (e.g., Besse and Courtillot, 2002). As moments of inertia depend on the degree-two spherical harmonic components (e.g., Lambeck, 1980), the estimates will not change in a major way even if we adopt other reconstruction models. The evalua- tion of TPW due to the northward drift of Australia, which substantially started at $\sim 40 \mathrm{Ma}$ (e.g., Golonka, 2002), may be a good example for examining the effects of continental drift on TPW (see Section 3.4). I then construct a hypothetical model for the distribution of continents and oceans at the present time by assuming that the continent including Australia at $40 \mathrm{Ma}$ is fixed during the past $40 \mathrm{Myr}$ and that distribution of other continents is the same as that for the present one. The configuration is illustrated in Fig. 3(g).

The surface area of the continents shown in Fig. 3 is different at different times. The number within the parenthesis for each figure indicates the percentage of the continental area to the area of the whole Earth, $A C(t)$. For example, 

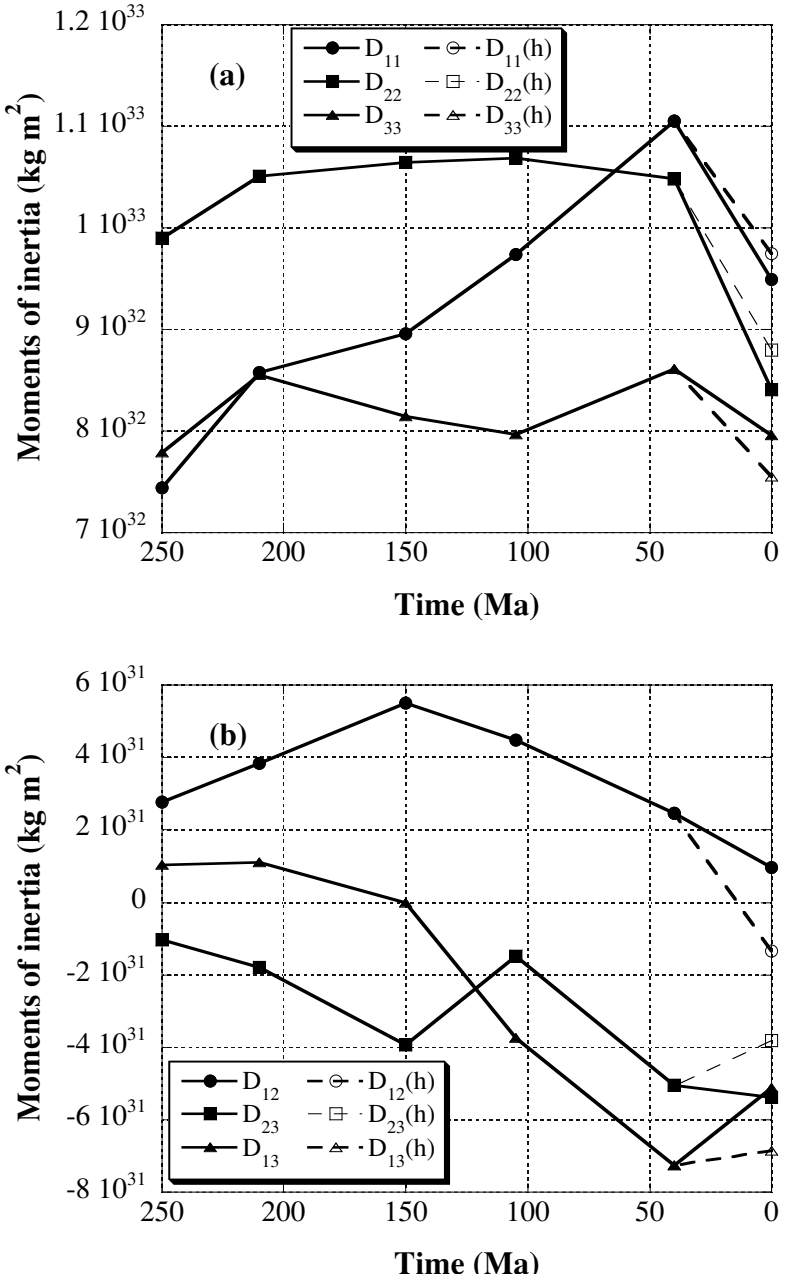

Fig. 4. Normalized moments of inertia, $D_{i j}(t) \times 35 / A C(t)$, for a density model given by Table 1 . The values of $A C(t)$ are shown in Fig. 3. The values denoted by $D_{i j}$ and $D_{i j}(\mathrm{~h})$ correspond to continental drift reconstructions by Condie and Sloan (1997) and the hypothetical model shown in Fig. 3(g), respectively.

the surface areas of the continents at 210 and 40 Ma cover 36.4 and $39.7 \%$ of the Earth's surface, respectively. However, the total land area above the present sea-level covers $\sim 30 \%$ of the Earth's surface, and the total continental area including continental shelves and slope covers $\sim 40 \%$ (e.g., Harrison et al., 1983). Here I assume the surface areas of continents to be $35 \%$ of the Earth's surface, and use the normalized moments of inertia, $D_{i j}(t) \times 35 / A C(t)$ (see Eq. (19)), to evaluate TPW. Figure 4 depicts the normalized time-dependent moment of inertia using the continental distributions and the density models shown in Fig. 2. In this study, I also evaluated TPW for models with $1.2 \times E_{i j}$ and $0.8 \times E_{i j}$ (see Eq. (18)) in order to examine the effects of the uncertainties of the density structures on TPW prediction. Whereas the magnitudes of TPW calculated from these models are different from the original one by about $20 \%$, the direction is identical to that of the original one.

\subsection{Effects of rotation axis and reference time on nu- merical results}

There are mainly two limitations in comparing the numerical results obtained here with geological evidence. One is the rotation axis for continental distribution and the other is the reference time for time integrations. I discuss these points in this section.

We illustrate the predictions by dimensionless quantities $m_{1}$ and $m_{2}$ defined by $\tan \left(m_{1}\right)=\omega_{1} / \omega_{3}$ and $\tan \left(m_{2}\right)=$ $\omega_{2} / \omega_{3}$, as used to illustrate TPW (Munk and MacDonald, 1960). The quantities $m_{1}$ and $m_{2}$ describe the displacement of the rotation axis in the directions $0^{\circ}$ and $90^{\circ} \mathrm{E}$, respectively. The values of $\omega_{1}, \omega_{2}$, and $\omega_{3}$ are solutions for the non-linear equations of Eqs. (5), (13) and (15). I therefore employ non-linear Liouville equations for all calculations and convert the TPW numbers into angular measures. Predictions are considered, for example, with a reference time of $210 \mathrm{Ma}$ for time integrations. The next step is to determine the distributions of continents and oceans defined for a rotation axis at $210 \mathrm{Ma}$ in solving the Liouville equation. However, I did not take into account the effects of TPW in modeling a continental drift model based on the reconstruction by Condie and Sloan (1997). That is, the present study implicitly assumes that the continental distributions after $210 \mathrm{Ma}$ are based on a reference frame of the rotation axis at $210 \mathrm{Ma}$. This assumption is obviously incorrect. However, I hopefully expect that the gross features of predicted TPW may provide some insights into the relationship between TPW and continental breakup and the following continental drift. For the past $40 \mathrm{Myr}$, however, the magnitude of TPW was significantly smaller than that of continental drift, particularly for the northward drift of Australia (e.g., Condie and Sloan, 1997; Besse and Courtillot, 2002). That is, the prediction may be insensitive to the rotation axis for the predictions with a reference time of $40 \mathrm{Ma}$.

For evaluating TPW owing to continental drift, it is necessary to adopt a reference time $\left(t_{0}\right)$ for time integrations $(t=0$ for Eqs. (5), (13) and (15)). The theoretical lower limit of the integration should be infinity to reduce the transient effects and to evaluate the viscoelastic responses accurately. In order to investigate the dependence on the start time of temporal integration practically, I adopted several reference times in evaluating TPW. Figures 5(a) and 5(b) show the predicted $m_{1}$ and $m_{2}$ for a plausible viscosity model of the mantle with $H=100 \mathrm{~km}, \eta_{\text {ltop }}=10^{24}$ and $\eta_{\operatorname{lm}}=10^{22}$ Pa s (e.g., Hager and Clayton, 1989; Nakada and Lambeck, 1989; Lambeck et al., 1990; Mitrovica and Forte, 2004). The reference times $\left(t_{0}\right)$ adopted here are 250, 210 and $40 \mathrm{Ma}$. The model results denoted by $m_{1}(210)$ and $m_{1}(210, \mathrm{~h})$, for example, indicate the predictions of $m_{1}$ with $t_{0}=210 \mathrm{Ma}$ for realistic and hypothetical continental drift models, respectively (see Fig. 3). To study the effect of different $t_{0}$, I also calculate TPW with $t_{0}=220$ and $230 \mathrm{Ma}$, with the initial continental distribution being the same as that of the realistic drift model at $210 \mathrm{Ma}$. The times for the distributions after $150 \mathrm{Ma}$ are the same as those in Fig. 3. The predictions are denoted by $m_{i}(220)$ and $m_{i}$ (230) for $i=1$ and 2 . The gross features for $t_{0}=250$ and $210 \mathrm{Ma}$ are relatively similar because the continental distribution remained quite stable for a period of 250-200 Ma (e.g., Condie and Sloan, 1997). This may mean that the standstill of TPW continued until $210 \mathrm{Ma}$. Although the predictions with $t_{0}=210,220$ and $230 \mathrm{Ma}$ are different from each other before $\sim 100 \mathrm{Ma}$, those after $\sim 100 \mathrm{Ma}$ are similar. 

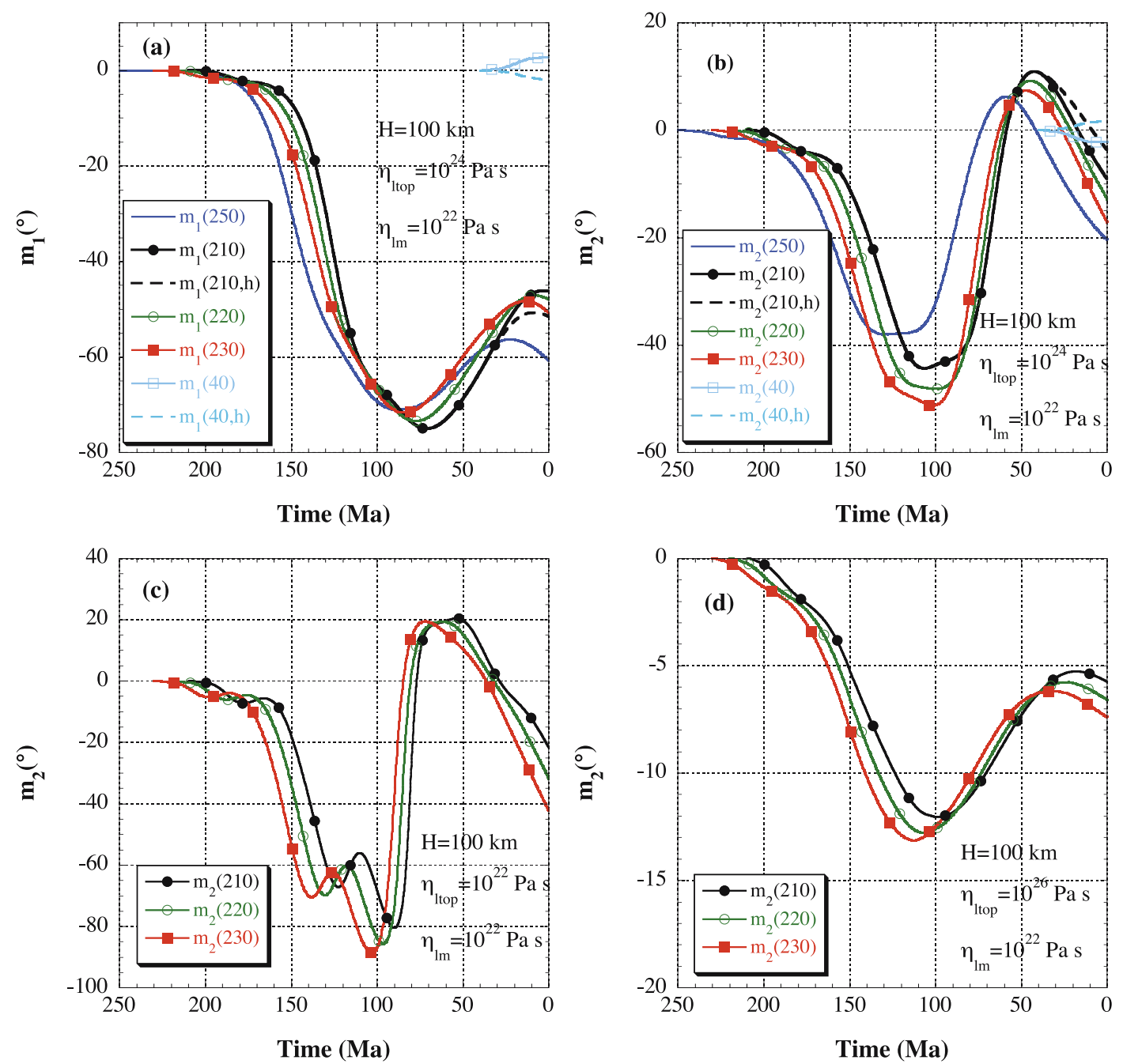

Fig. 5. (a), (b) Predicted $m_{1}$ and $m_{2}$ for a viscosity model with $H=100 \mathrm{~km}, \eta_{\mathrm{ltop}}=10^{24}$ and $\eta_{\mathrm{lm}}=10^{22}$ Pa s. The reference times $\left(t_{0}\right)$ adopted here are 250,210,220, 230 and $40 \mathrm{Ma}$. The model results denoted by $m_{1}(210)$ and $m_{1}(210, \mathrm{~h})$, for example, indicate the predictions of $m_{1}$ with $t_{0}=210 \mathrm{Ma}$ for realistic and hypothetical continental drift models, respectively. See text for predictions for $m_{i}(220)$ and $m_{i}(230)$ with $i=1$ and 2 . (c) Predicted $m_{2}$ for a viscosity model with $H=100 \mathrm{~km}, \eta_{\mathrm{ltop}}=10^{22}$ and $\eta_{\mathrm{lm}}=10^{22} \mathrm{~Pa} \mathrm{~s}$, and (d) for $H=100 \mathrm{~km}, \eta_{\mathrm{ltop}}=10^{26}$ and $\eta_{\mathrm{lm}}=10^{22} \mathrm{~Pa}$

This may imply that a warm-up period of $\sim 10-20$ Myr is required to reduce transient effects and to obtain physically meaningful polar wander. Figures 5(c) and 5(d) depict the results with $\eta_{\text {ltop }}=10^{22}$ and $\eta_{\text {ltop }}=10^{26} \mathrm{~Pa} \mathrm{~s}$, respectively, which also support the results obtained from the predictions with $\eta_{\text {ltop }}=10^{24} \mathrm{~Pa}$ s. That is, it would be possible to examine the effects of continental drift on TPW at least after $\sim 130 \mathrm{Ma}$ based on the predictions with $t_{0}=210 \mathrm{Ma}$.

\subsection{Effects of northward drift of Australia on TPW}

Evaluation of TPW due to the northward drift of Australia is a good example for examining the effects of continental drift on TPW. It is important to note that the central latitude of Australia at $\sim 40 \mathrm{Ma}$ was $\sim 45^{\circ} \mathrm{S}$ (e.g., Condie and Sloan, 1997; Golonka, 2002) and that Australia was located approximately on the antipode of Greenland to the center of the Earth at $\sim 40 \mathrm{Ma}$ (see Fig. 3(e)). In fact, seafloor spreading between Australia and Antarctica accelerated to $\sim 20 \mathrm{~mm} \mathrm{yr}^{-1}$ at $\sim 40 \mathrm{Ma}$ and the substantial northward drift of Australia started at $\sim 40 \mathrm{Ma}$ (e.g., Golonka, 2002). In the TPW associated with continental drift examined here, the northward drift along the meridian of lon- gitude including Australia and Greenland inevitably causes the polar wander direction towards Greenland because the rotation axis wanders towards the maximum principal axis of inertia (Munk and MacDonald, 1960).

In examining the effects of northward drift of Australia on TPW, it is required that the predictions for the past $\sim 40 \mathrm{Myr}$ with $t_{0}=210 \mathrm{Ma}$ are similar to those with, for example, $t_{0}=105 \mathrm{Ma}$. The predictions are, however, different each other because of the difference in the reference axis. Here I examine the difference in the predictions between realistic and hypothetical continental drift models. Figure 6(a) shows the difference in the predicted TPW for these drift models, for example, $m_{1}(210, \mathrm{r}-\mathrm{h})$ given by $m_{1}(210)-m_{1}(210, \mathrm{~h})$. The predictions for $t_{0}=210$ and $230 \mathrm{Ma}$ are very similar as was explained above. The predictions for $t_{0}=40$ and $250 \mathrm{Ma}$, in which the reference axes are different from that for $t_{0}=210$ and $230 \mathrm{Ma}$, are not so different from those with $t_{0}=210$ and $230 \mathrm{Ma}$. That is, the difference shown in Fig. 6(a) is relatively insensitive to the reference time adopted in the calculations. However, it should be noted that the predicted TPW during the past 40 

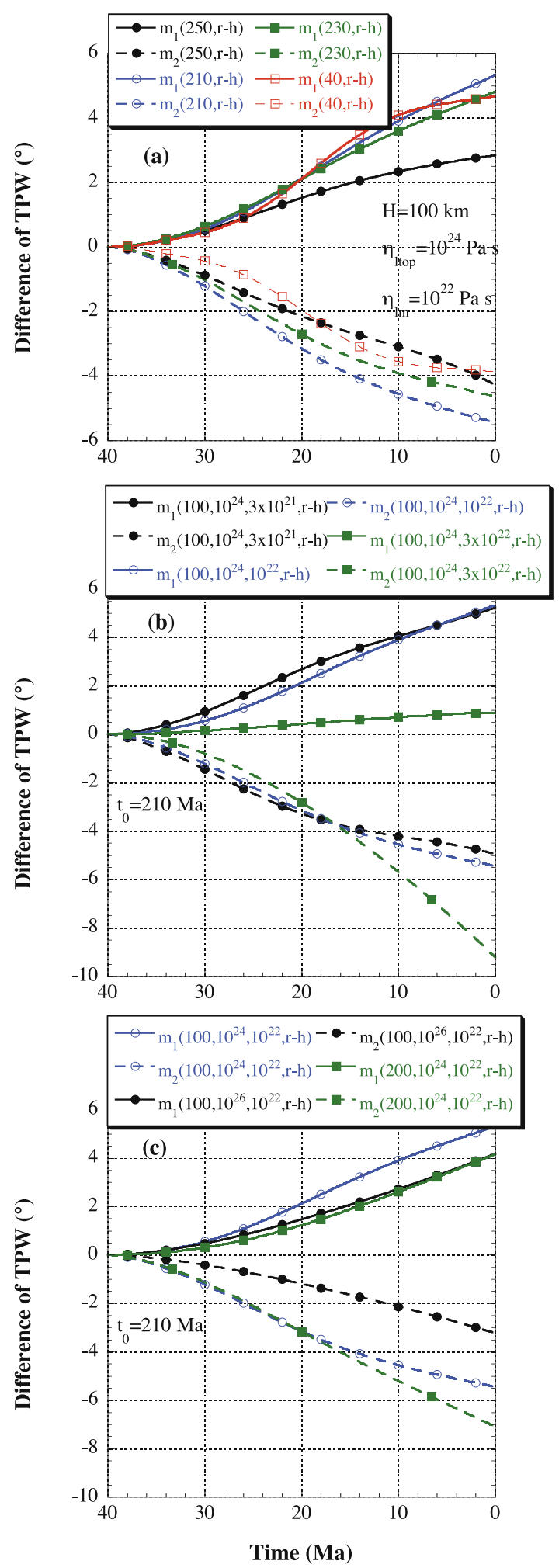

Fig. 6. (a) Difference in the predicted TPW between realistic and hypothetical continental drift models: for example, $m_{1}(210, \mathrm{r}-\mathrm{h})$ given by $m_{1}(210)-m_{1}(210, \mathrm{~h})$, for a viscosity model with $H=100 \mathrm{~km}$, $\eta_{\text {ltop }}=10^{24}$, and $\eta_{\mathrm{lm}}=10^{22} \mathrm{~Pa}$ s. The reference times $\left(t_{0}\right)$ adopted here are 250, 210, 220, 230 and $40 \mathrm{Ma}$ (see Fig. 5). (b), (c) Difference in TPW between realistic and hypothetical continental drift models (see Fig. 3) for a reference time of $t_{0}=210 \mathrm{Ma}$. The model results by $m_{1}\left(100,10^{24}, 10^{22}, \mathrm{r}-\mathrm{h}\right)$, for example, indicate the prediction for $m_{1}$ with $t_{0}=210 \mathrm{Ma}, H=100 \mathrm{~km}, \eta_{\mathrm{ltop}}=10^{24}$, and $\eta_{\mathrm{lm}}=10^{22} \mathrm{~Pa} \mathrm{~s}$.

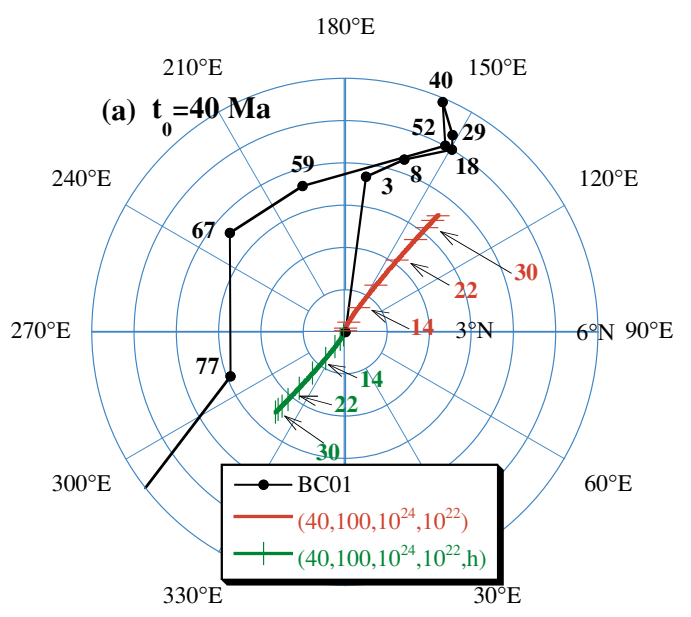

$0^{\circ} \mathrm{E}$

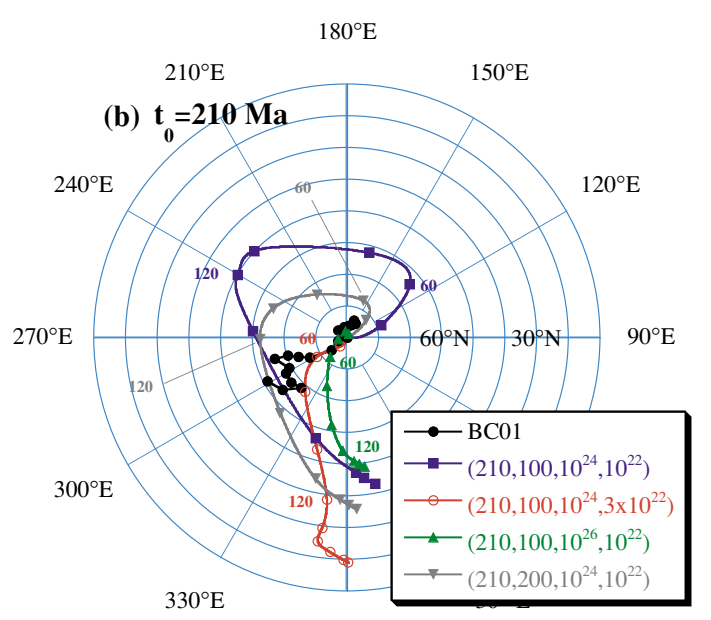

$0^{\circ} \mathrm{E}$

$180^{\circ} \mathrm{E}$

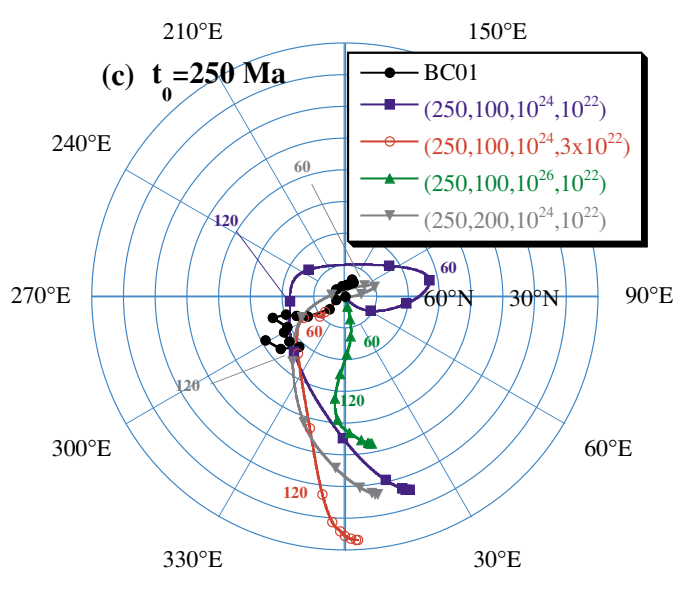

$0^{\circ} \mathrm{E}$

Fig. 7. Observed and predicted TPW paths with time unit of Ma. (a) Observed TPW path inferred from paleomagnetic data (Besse and Courtillot, 2002), referred to as $\mathrm{BC} 01$, and predictions for a model with $t_{0}=40 \mathrm{Ma}, H=100 \mathrm{~km}, \eta_{\mathrm{ltop}}=10^{24}$, and $\eta_{\mathrm{lm}}=10^{22} \mathrm{~Pa}$ $\mathrm{s}$. The model results denoted by $\left(40,100,10^{24}, 10^{22}\right)$, relative to the present rotation axis, are based on the continental distributions shown for Figs. 3(e) and 3(f) and those denoted by $\left(40,100,10^{24}, 10^{22}, \mathrm{~h}\right)$ are for Fig. 3(e) and a hypothetical continental distribution of Fig. 3(g). Tickmarks for each predicted TPW are indicated at 4-Myr intervals. (b), (c) Observed TPW path and predicted TPW paths for models with $t_{0}=210$ and $250 \mathrm{Ma}$ and several viscosity models. Model results denoted by $\left(210,200,10^{24}, 10^{22}\right)$, for example, indicate the predictions with $t_{0}=210 \mathrm{Ma}, H=200 \mathrm{~km}, \eta_{\text {ltop }}=10^{24}$ and $\eta_{\mathrm{lm}}=10^{22} \mathrm{~Pa}$ s. Symbols for each predicted TPW are indicated at 20-Myr intervals. 


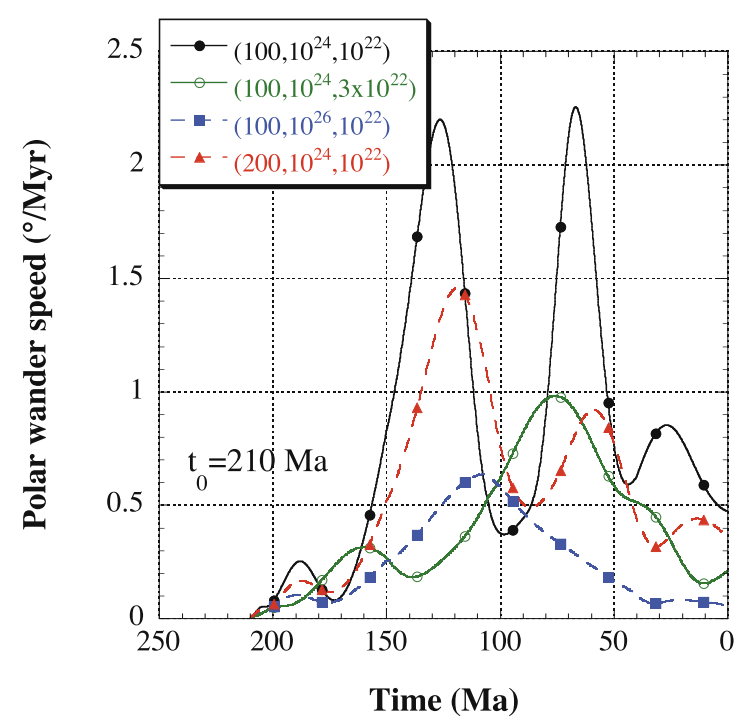

Fig. 8. Predicted TPW speeds for models with $t_{0}=210$ and several viscosity models. Model results denoted by $\left(200,10^{24}, 10^{22}\right)$, for example, indicate the predictions with $t_{0}=210 \mathrm{Ma}, H=200 \mathrm{~km}, \eta_{\text {ltop }}=10^{24}$ and $\eta_{\mathrm{lm}}=10^{22} \mathrm{~Pa} \mathrm{~s}$.

Myr relative to the present-day value is predominantly contributed by the viscoelastic responses to temporal changes in moments of inertia for continental drift before $40 \mathrm{Ma}$ (see Fig. 5). That is, it is difficult to discuss TPW itself associated with continental drift with $t_{0}=40 \mathrm{Ma}$.

In order to examine the potential effects of the northward drift of Australia on TPW, Figs. 6(b) and 6(c) depict the difference of TPW between realistic and hypothetical continental drift models (see Fig. 3) for a reference time of $t_{0}=$ $210 \mathrm{Ma}$. The model results by $m_{1}\left(100,10^{24}, 10^{22}, \mathrm{r}-\mathrm{h}\right)$, for example, indicate the prediction for $m_{1}$ with $t_{0}=210 \mathrm{Ma}$, $H=100 \mathrm{~km}, \eta_{\mathrm{ltop}}=10^{24}$ and $\eta_{\mathrm{lm}}=10^{22} \mathrm{~Pa} \mathrm{~s}$. These predictions indicate that the predicted TPW is sensitive to the lithospheric and lower mantle viscosities. The temporal changes in moments of inertia induce TPW with a magnitude larger than $5^{\circ}$ during the past $40 \mathrm{Myr}$ regardless of the viscosity models.

\subsection{Predicted TPW paths and polar wander speeds due to continental drift}

In this section, we examine the predicted TPW paths relative to the present rotation axis. Figure 7(a) illustrates the predictions with $t_{0}=40 \mathrm{Ma}$ for a plausible viscosity model with $H=100 \mathrm{~km}, \eta_{\text {ltop }}=10^{24}$ and $\eta_{\mathrm{lm}}=10^{22} \mathrm{~Pa} \mathrm{~s}$, denoted by $\left(40,100,10^{24}, 10^{22}\right)$, and also the predictions for the hypothetical drift model denoted by $\left(40,100,10^{24}, 10^{22}, \mathrm{~h}\right)$. Tickmarks for predicted TPW are indicated at 4-Myr intervals. The direction for $\left(40,100,10^{24}, 10^{22}, \mathrm{~h}\right)$ is opposite to that for $\left(40,100,10^{24}, 10^{22}\right)$, which is consistent with the qualitative estimate of TPW owing to northward drift of Australia. Figure 7(a) also illustrates the TPW path inferred from paleomagnetic data by Besse and Courtillot (2002) under the hypothesis of fixed Atlantic and Indian hotspots. Whereas the prediction for $\left(40,100,10^{24}, 10^{22}\right)$ is relatively similar to the observation, polar wander predicted with, for example, $t_{0}=210 \mathrm{Ma}$ is much more reliable as stated in Section 3.3.
In order to compare the predictions for $t_{0}=210$ and 250 Ma with observations, I illustrate the predicted TPW paths relative to the present rotation axis in Figs. 7(b) and 7(c). The present study is, however, preliminary because of an implicit assumption for the reference axis at $210 \mathrm{Ma}$ as stated in Section 3.3. The gross features of these predictions are similar to each other because the continental distribution remained quite stable for a period of 250 to $200 \mathrm{Ma}$ (e.g., Condie and Sloan, 1997) and may provide a possible relationship between TPW and the continental breakup of Pangea followed by seafloor spreading of the Atlantic Ocean. The predicted TPW path changes systematically with increasing lithospheric and lower mantle viscosities. The predictions prior to $130 \mathrm{Ma}$ are significantly different from the observations regardless of the viscosity models adopted here. The observed gross features after 130 Ma may be explained by the predictions for an earth model with, for example, $10^{22}<\eta_{\mathrm{lm}}<3 \times 10^{22} \mathrm{~Pa}$ s for $\eta_{\text {ltop }}=10^{24} \mathrm{~Pa}$ s as inferred from the predicted TPW paths for $\eta_{\mathrm{lm}}=10^{22}$ and $3 \times 10^{22} \mathrm{~Pa} \mathrm{~s}$, whereas the predictions for $\eta_{\mathrm{lm}} \geq 3 \times 10^{22} \mathrm{~Pa}$ s and $\eta_{\text {ltop }} \sim 10^{26} \mathrm{~Pa}$ s cannot explain the change of TPW direction at $\sim 40 \mathrm{Ma}$.

Figure 8 depicts the results for predicted TPW speeds with $t_{0}=210 \mathrm{Ma}$ for several viscosity models. The peak positions shift to a younger age and the magnitude decreases with increasing lower mantle viscosity as clearly indicated by the predictions of $\left(100,10^{24}, 10^{22}\right)$ and $\left(100,10^{24}, 3 \times 10^{22}\right)$. However, sharp peaks are not predicted for an earth model with a depth-dependent viscosity model with $\eta_{\text {ltop }}=10^{26} \mathrm{~Pa}$ s. The gross trend between 130 and 50 Ma may be predominantly be related to continental breakup of Pangea and the following continental drift associated with seafloor spreading of the Atlantic Ocean. In a model of $\left(200,10^{24}, 10^{22}\right)$, for example, the predicted average polar wander rates for $130-50 \mathrm{Ma}$ are $\sim 0.8^{\circ} \mathrm{Myr}^{-1}$, which is rather consistent with the observations of $0.3^{\circ}-0.5^{\circ}$ $\mathrm{Myr}^{-1}$ (Besse and Courtillot, 2002). Those for 50-10 Ma are $\sim 0.4^{\circ} \mathrm{Myr}^{-1}$ and are smaller than those for $130-50 \mathrm{Ma}$.

\section{Conclusions}

Convective processes in the mantle such as downgoing slabs and upwelling plumes are undoubtedly essential factors causing continental drift and long-term polar wander (Ricard and Sabadini, 1990; Spada et al., 1992; Ricard et al., 1993; Richards et al., 1997, 1999; Steinberger and O'Connell, 1997, 2002; Greff-Lefftz, 2004). However, the present study suggests that temporal changes in moments of inertia due to continental drift also have a significant effect on TPW. That is, TPW throughout the Cenozoic and Mesozoic times may be attributed to the time-dependent continental distribution associated with the breakup of Pangea followed by seafloor spreading of the Atlantic Ocean. It is therefore important to model TPW to include the effects of both the driving forces such as mantle convection and lithosphere subduction and the isostatically compensated surface plate motions.

Acknowledgments. I thank S. Karato, H. Sano and S. Kaneshima for their valuable comments and discussion and the editor (Takeshi Sagiya) for his efforts. Constructive comments by Y. Hamano, 
B. Steinberger and S. Dickman were very helpful in improving the paper. This work was supported by the Japanese Ministry of Education, Science and Culture (Grand-in-Aid for Scientific Research No. 17340132 and 17654088).

\section{References}

Besse, J. and V. Courtillot, Revised and synthetic apparent polar wander paths of the African, Eurasian, North American and Indian plates, and true polar wander since $200 \mathrm{Ma}$, J. Geophys. Res., 96, 4029-4050, 1991.

Besse, J. and V. Courtillot, Apparent and true polar wander and the geometry of the geomagnetic field over the last $200 \mathrm{Myr}, J$. Geophys. Res., 107(B11), 2300, doi: 10.1029/2000JB000050, 2002.

Condie, K. C. and R. E. Sloan, Origin and Evolution of Earth: Principles of Historical Geology, Prentice Hall, New Jersey, 1997.

Dickman, S. R., Secular trend of the Earth's rotation pole: Consideration of motion of the latitude observatories, Geophys. J. R. Astr. Soc., 51, 229-244, 1977.

Dickman, S. R., Continental drift and true polar wandering, Geophys. J. R. Astr. Soc., 57, 41-50, 1979.

Dziewonski, A. M. and D. L. Anderson, Preliminary reference Earth model (PREM), Phys. Earth Planet. Inter., 25, 297-356, 1981.

Gold, T., Instability of the Earth's axis of rotation, Nature, 175, 526-529, 1955.

Goldreich, P. and A. Toomre, Some remarks on polar wandering, J. Geophys. Res., 74, 2555-2567, 1969.

Golonka, J., Plate-tectonic maps of the Phanerozoic, Phanerozoic Reef Patterns, in SEPM Special Publication No.72, edited by W. Kiessling, E. Flügel and J. Golonka, pp. 21-75, Society for Sedimentary Geology, Tulsa, Oklahoma, USA, 2002.

Greff-Lefftz, M., Upwelling plumes, superwells and true polar wander, Geophys. J. Int., 159, 1125-1137, 2004.

Hager, B. H. and R. W. Clayton, Constraints on the structure of mantle convection using seismic observations, flow models, and the geoid, in Mantle Convection: Plate Tectonics and Global Dynamics, edited by W. R. Peltier, pp.657-763, Gordon and Breach Science Publications, New York, 1989.

Harrison, C. G. A., K. J. Miskell, G. W. Brass, E. S. Saltzman, and J. L. Sloan II, Continental hypsography, Tectonics, 2, 357-377, 1983.

Johnston, P. and K. Lambeck, Postglacial rebound and sea level contributions to changes in the geoid and the Earth's rotation axis, Geophys. $J$. Int., 136, 537-558, 1999.

Lambeck, K., The Earth's Variable Rotation: Geophysical Causes and Consequences, Cambridge University Press, Oxford, 1980.

Lambeck, K., P. Johnston, and M. Nakada, Holocene glacial rebound and sea-level change in NW Europe, Geophys. J. Int., 103, 451-468, 1990.

Lemoine, F. G., S. C. Kenyon, J. K. Factor, R. G. Trimmer, N. K. Palvis, D. S. Chinn, C. M. Cox, S. M. Klosko, S. B. Luthcke, M. H. Torrence, Y. M. Wang, R. G. Williamson, E. C. Palvis, R. H. Rapp, and T. R. Olson, The development of the Joint NASA GSFC and the National Imagery and Mapping Agency (NIMA) Geopotential Model EGM96, Technical report, NASA/TP-1998-206861, 1998.

McCarthy, D. D. and B. J. Luzum, Path of the mean rotational pole from 1899 to 1994, Geophys. J. Int., 12, 623-629, 1996.

Minster, J. B., T. H. Jordan, P. Molnar, and E. Haines, Numerical modelling of instantaneous plate tectonics, Geophys. J. R. Astr. Soc., 36, 541-576, 1974.

Mitrovica, J. X. and A. M. Forte, A new inference of mantle viscosity based upon joint inversion of convection and glacial isostatic adjustment data, Earth Planet. Sci. Lett., 225, 177-189, 2004.

Mitrovica, J. X. and G. A. Milne, Glaciation-induced perturbations in the Earth's rotation: A new appraisal, J. Geophys. Res., 103, 985-1005, 1998.
Mitrovica, J. X., J. Wahr, I. Matsuyama, and A. Paulson, The rotational stability of an ice-age earth, Geophys. J. Int., 161, 491-506, 2005.

Munk, W. H. and G. J. F. MacDonald, The Rotation of the Earth: a Geophysical Discussion, Cambridge Univ. Press, Cambridge, 1960.

Nakada, M., Polar wander caused by the Quaternary glacial cycles and fluid Love number, Earth Planet. Sci. Lett., 200, 159-166, 2002.

Nakada, M. and K. Lambeck, Late Pleistocene and Holocene sea-level change in the Australian region and mantle viscosity, Geophys. J., 96, 497-517, 1989.

Nakada, M. and J. Okuno, Perturbations of the Earth's rotation and their implications for the present-day mass balance of both polar ice caps, Geophys. J. Int., 152, 124-138, 2003.

Peltier, W. R., The impulse of a Maxwell Earth, Rev. Geophys. Space Phys., 12, 649-669, 1974

Peltier, W. R., Glacial isostatic adjustment II, the inverse problem, Geophys. J. R. Astr. Soc., 46, 669-706, 1976.

Prévot, M., E. Mattern, P. Camps, and M. Daignières, Evidence for a $20^{\circ}$ tilting of the Earth's rotation axis 110 million years ago, Earth Planet. Sci. Lett., 179, 517-528, 2000.

Ricard, Y. and R. Sabadini, Rotational instabilities of the Earth induced by mantle density anomalies, Geophys. Res. Lett., 17, 627-630, 1990.

Ricard, Y., G. Spada, and R. Sabadini, Polar wandering of a dynamic earth, Geophys. J. Int., 113, 284-298, 1993.

Richards, M. A., Y. Ricard, C. Lithgow-Bertelloni, G. Spada, and R. Sabadini, An explanation for Earth's long-term rotational stability, Science, 297, 372-375, 1997.

Richards, M. A., H. P. Bunge, Y. Ricard, and J. R. Baumgardner, Polar wandering in mantle convection models, Geophys. Res. Lett., 26, 1777$1780,1999$.

Sabadini, R. and W. R. Peltier, Pleistocene deglaciation and the Earth's rotation: Implications for mantle viscosity, Geophys. J. R. Astr. Soc., 66 , 553-578, 1981.

Spada, G., Y. Ricard, and R. Sabadini, Excitation of true polar wander by subduction, Nature, 360, 452-454, 1992.

Steinberger, B. M. and R. J. O'Connell, Changes of the Earth's rotation axis inferred from advection of mantle density heterogeneities, Nature, 387, 169-173, 1997.

Steinberger, B. M. and R. J. O'Connell, The convective mantle flow signal in rates of true polar wander, Ice Sheets, Sea Level and Dynamic Earth, in Geodynamics Series 29, edited by J. X. Mitrovica and L. L. A. Vermeersen, pp. 233-256, American Geophysical Union, Washington, DC, 2002.

Tarduno, J. A., R. A. Duncan, D. W. Scholl, R. C. Cottrell, B. Steinberger, T. Thordarson, B. C. Kerr, C. R. Neal, F. A. Frey, M. Torii, and C. Carvallo, The Emperor Seamounts: southward motion of the Hawaiian hotspot plume in Earth's mantle, Science, 301, 1064-1069, 2003.

Turcotte, D. L. and G. Schubert, Geodynamics, Cambridge University Press, Cambridge, 2002.

Vermeersen, L. L. A. and N. J. Vlaar, Changes in the Earth's rotation by tectonic movements, Geophys. Res. Lett., 20, 81-84, 1993.

Vermeersen, L. L. A., R. Sabadini, G. Spada, and N. J. Vlaar, Mountain building and Earth rotation, Geophys. J. Int., 117, 610-624, 1994.

Vermeersen, L. L. A. and R. Sabadini, Significance of the fundamental mantle relaxation mode in polar wander simulations, Geophys. J. Int., 127, F5-F9, 1996.

Watts, A. B., Isostasy and Flexure of the Lithosphere, Cambridge Univ. Press, Cambridge, 2001

$\mathrm{Wu}, \mathrm{P}$. and W. R. Peltier, Pleistocene deglaciation and the Earth's rotation: A new analysis, Geophys. J. R. Astr. Soc., 76,753-791, 1984.

M. Nakada (e-mail: mnakada@geo.kyushu-u.ac.jp) 